\title{
Research on isomer decay with the NEPTUNE spectrometer
}

\author{
S. Oberstedt ${ }^{1, \text { a }}$, A. Oberstedt ${ }^{2}$, A. Plompen ${ }^{1}$, V. Semkova ${ }^{1}$, G. Lövestam ${ }^{1}$, and M. Gawrys ${ }^{3}$ \\ ${ }^{1}$ EC-JRC Institute for Reference Materials and Measurements, 2440 Geel, Belgium \\ 2 Department of Natural Sciences, Örebro University, 70182 Örebro, Sweden \\ 3 Department of Fundamental Physics, Chalmers University of Technology, 41296 Göteborg, Sweden
}

\begin{abstract}
A new spectrometer has been built at the EC-JRC IRMM to investigate isomer decay in the millisecond range and activation cross sections of isotopes, where isomeric states exist and are populated. The spectrometer is equipped with high-resolution gamma-ray detectors and an ionisation chamber for the measurement of charged particles, e.g., fission fragments. NEPTUNE provides pulsed quasi mono-energetic neutrons at pulse repetition frequencies up to $5 \mathrm{kHz}$ and tuneable neutron pulse widths ranging from $10 \mu$ s into the ms-region. For this purpose a beam chopper based on a parallel-plate capacitor has been integrated into the accelerator beam line in order to deflect the charged-particle beam onto a tantalum beam dump. First research has been performed with the NEPTUNE spectrometer dedicated to the shape isomer search in the odd-A uranium isotopes ${ }^{235} \mathrm{U},{ }^{237} \mathrm{U}$ and ${ }^{239} \mathrm{U}$. In further experiments the population of long-lived spin isomers was investigated.
\end{abstract}

\section{Introduction}

The picture of a double-humped barrier in the nuclear energy landscape is able to successfully explain a number of phenomena revealed in nuclear reactions on actinide nuclei, see e.g., refs. [1-3]. The observed intermediate structure in sub-threshold fission as well as meta-stable fission isomers may be described within this model. Decay mode and half-life of these so-called shape isomers directly probe the fission barrier height and penetrability, which both are important input parameters for the modelling of nuclear reactions.

Since the early 1960s many fission isomers have been discovered in nuclei ranging from ${ }^{234} \mathrm{U}$ to ${ }^{245} \mathrm{Bk}$ [4]. One of the persisting problems associated with fission isomers is the interesting question of competing decay modes as for example decay by $\gamma$-ray emission, which has been studied thoroughly in the 1980s and 1990s in ${ }^{240} \mathrm{Pu}$ [5], ${ }^{238} \mathrm{U}$ [6] and ${ }^{236} \mathrm{U}$ [7]. Even more intriguing today is the lack of any shape-isomer half-life data for odd-N uranium and neptunium isotopes. Only for ${ }^{239} \mathrm{U}$ the population of the super-deformed (SD) ground state in a neutron-induced capture experiment was observed so far [8,9]. Since for these isotopes fission half-lives are expected to be of the order of several hundreds of $\mu \mathrm{s}$ or even longer, the detection with commonly used pulsed particle beams is very difficult. It is even more difficult in neutroninduced reactions, where the environmental background created from subsequent pulses is extremely disadvantageous. Together with the extremely low production cross sections for shape isomers, typically of the order of a few $\mu \mathrm{b}$, as well as half-life predictions ranging in some cases over five orders of magnitude, leaves the measurement of shape-isomer decay data to a challenging venture for the experimentalist.

Here, we report about the construction of a dedicated isomer spectrometer and present results from first experiments, performed during the first two years of operation.

\footnotetext{
${ }^{a}$ Presenting author, e-mail: stephan .oberstedt@ec. europa . eu
}

\section{The isomer spectrometer NEPTUNE}

In the frame of the EC-JRC exploratory research exercise 2004, the design and construction of the NEPTUNE (NEw Pulsed and TUneable NEutron source) isomer spectrometer had been started [10]. NEPTUNE stands for the neutron source coupled to various particle detectors. For this purpose a new pulsing device was integrated into one of the Van de Graaff (VdG) accelerator beam lines. The purpose was to produce pulsed neutron beams with variable duty cycles and pulse lengths for investigations of activation products with half-lives below $1 \mathrm{~s}$, meta-stable states in fission-fragments and shortlived super-deformed (SD) shape isomers in actinide nuclei. NEPTUNE provides pulsed quasi mono-energetic neutron beams with repetition rates between less than $1 \mathrm{~Hz}$ and $5 \mathrm{kHz}$ with a pulse width tuneable between $10 \mu$ s and the maximum length between two successive neutron pulses. The principle is based on the deflection of the charged-particle beam before hitting the neutron production target. The deflection system consists of a pair of parallel steering plates to which a high voltage is applied. The high voltage, powered by a high voltage pulse generator [11], is adjusted according to the particle energy to deflect the beam onto a tantalum beam dump at about $3 \mathrm{~m}$ distance from the deflection device and is driven by a precision pulse generator with tuneable pulse repetition frequency and length. Tantalum is chosen as beam dump material because of its high melting point of higher than $3300 \mathrm{~K}$, a value only exceeded by tungsten and rhenium, and its very low $(\mathrm{p}, \mathrm{xn})$ cross section at proton energies below $4.5 \mathrm{MeV}, \sigma_{p n} \leq 1.1 \times 10^{-4} \mathrm{~b}$ [12]. Furthermore, the module provides a trigger signal to give full control on the timing between the start of a neutron pulse and a registered detector event. In the upper part of figure 1 a photograph of the accelerator beam line with the pair of electric steering plates is shown. The NEPTUNE spectrometer may be equipped with different detector systems according to the requirements of a particular experiment. Ionisation chambers are used to 

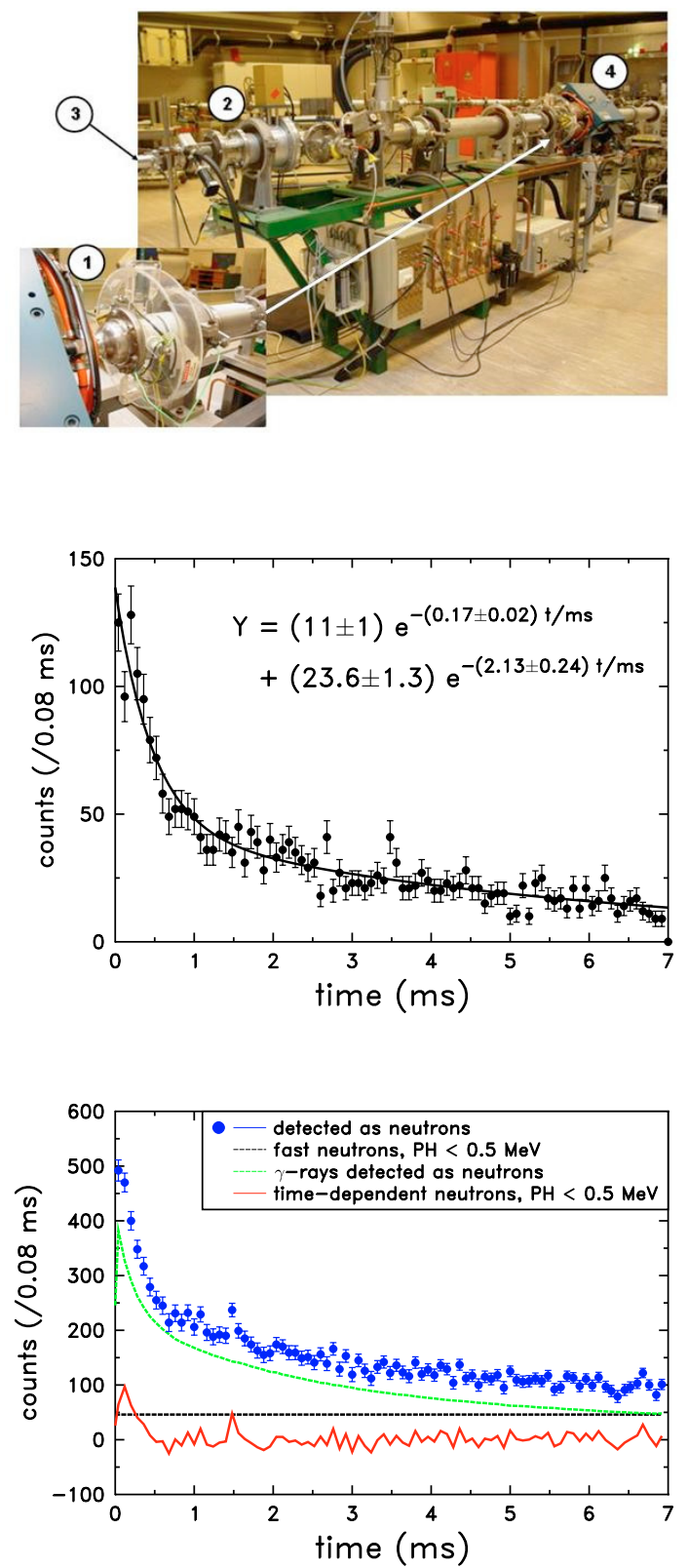

Fig. 1. Top: NEPTUNE isomer spectrometer: (1) pair of electrostatic steering plates, (2) Ta beam dump, (3) neutron source, (4) focussing magnet; middle: time distribution of fission events from the reaction ${ }^{235} \mathrm{U}(\mathrm{n}, \mathrm{f})$ induced by thermalised neutrons within $41.4 \mathrm{~h}$; bottom: corresponding time distribution of events detected as fast neutrons in the liquid scintillation detector.

investigate shape-isomeric fission and to monitor residual low-energy neutron background. High-resolution $\gamma$-ray detectors behind appropriate radiation shielding are used to investigate de-excitation of (shape-) isomers through $\gamma$-ray emission.

During an experiment the neutron background due to scattered neutrons, moderated to thermal energies typically within some tens of $\mu \mathrm{s}$, is monitored with a sample of fissile material, here about $2.4 \mathrm{mg}$ of ${ }^{235} \mathrm{U}$ (middle part of fig. 1). A NE213-equivalent scintillation detector, capable to discriminate between neutrons and $\gamma$-rays, is placed at $0^{\circ}$ relative to the neutron beam-axis to monitor the quality of the proton beam deflection. A reduction of the neutron flux in beam-OFF position by a factor of $10^{7}$ may be achieved.

\section{First experimental results}

The first experiments were dedicated to investigate the decay of the SD shape isomer in odd-A uranium isotopes. $I n{ }^{239} \mathrm{U}$ the possible population of the shape isomer has been observed and the corresponding ground state energy, $\mathrm{E}_{I I}$, was determined previously $[8,9]$. However, no subsequent decay by either fission or $\gamma$-decay back to the normal ground state was found, yet. If the half-lives range from several tens of $\mu$ s to several ms as predicted, this decay should be found with NEPTUNE. Next, the fission decay mode of a possible shape isomer in ${ }^{235} \mathrm{U}$ has been searched for, whose half-life is estimated to lie between 1 and $10 \mathrm{~ms}$ [14-16]. In the meantime, the range of applications of the NEPTUNE spectrometer has been extended by a feasibility study of spin-isomer population and subsequent de-excitation by $\gamma$-ray emission in ${ }^{206} \mathrm{~Pb}$. This particular delayed reaction process is important to determine correct cross section data for inelastic neutron scattering reactions, which are relevant for lead-cooled reactors and subcritical reactor assemblies.

\subsection{Spin-isomer population cross sections in lead isotopes}

This experiment has been performed in the context of a measurement series based on the (n, xn) technique at the GELINA facility [13]. In the course of this work it became clear, that for two important lead isotopes spin isomer population plays an important role. These isomers cannot be tackled by the technique employed at GELINA. One relevant isomer is the $11^{\text {th }}$ excited state of ${ }^{206} \mathrm{~Pb}\left(\mathrm{E}^{*}=2200 \mathrm{keV}, \mathrm{J}^{\pi}=13 / 2^{+}\right.$, $\mathrm{T}_{1 / 2}=125 \mu \mathrm{s}$ ). This isomer carries an important fraction of the inelastic cross section and, therefore, is of importance to verify model predictions with experimental data. The purpose of this experiment was to demonstrate NEPTUNE's ability to clearly identify $\gamma$-rays from this decay and to determine its contribution to the cross section due to the de-population of the isomer state. Experiments were performed with a repetition frequency $v=(750 \mu \mathrm{s})^{-1}$ and a neutron pulse width of $250 \mu \mathrm{s}$. The incident neutron energies were $\mathrm{E}_{n}=5.1,6.2$ and $7 \mathrm{MeV}$. As shown in the top part of figure 2, the relevant $\gamma$-peak at $\mathrm{E}_{\gamma}=803 \mathrm{keV}$ has been clearly identified. The calculated decay curve describes well the time spectrum constructed for $\mathrm{E}_{\gamma}=803 \mathrm{keV}$ (bottom part of fig. 2, lower full line) and demonstrates the feasibility to obtain correct data on spinisomer population with NEPTUNE.

\subsection{Investigation of the $\gamma$-decay of the super-deformed ground state in ${ }^{239} \mathrm{U}$}

The experiment was performed with NEPTUNE settings of 1 and $5 \mathrm{kHz}$ with neutron pulse lengths of $300 \mu \mathrm{s}$ and $60 \mu \mathrm{s}$, respectively. The sample consisted of $157 \mathrm{~g}$ depleted uranium, contained in a brass can with thin $(0.3 \mathrm{~mm})$ aluminium windows. The sample diameter was $115 \mathrm{~mm}$. The can was centred in an aluminium frame at the same height as the 

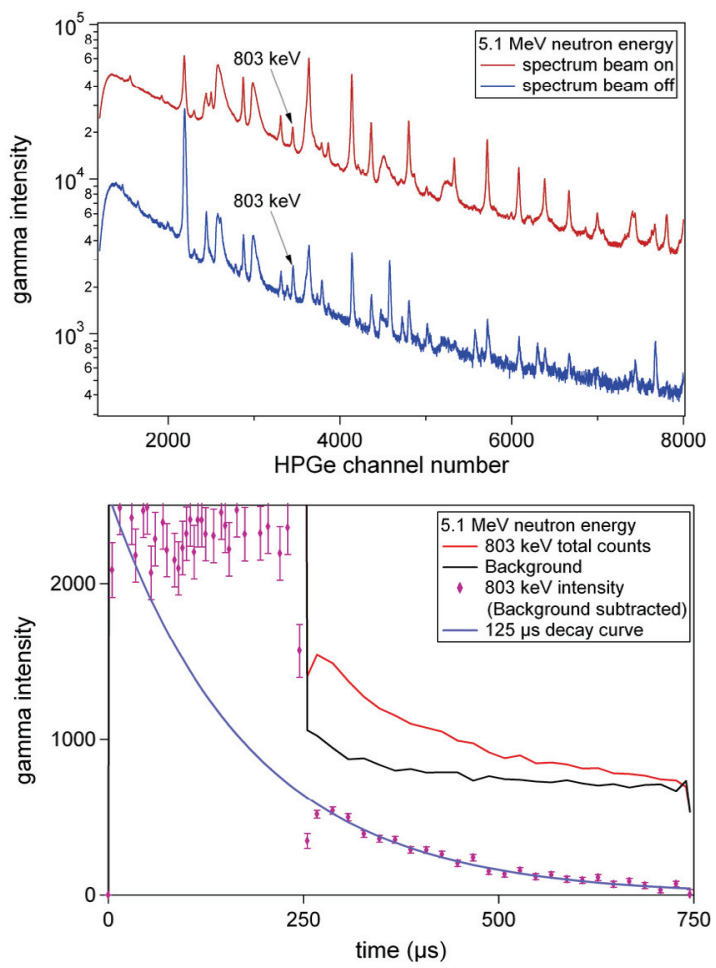

Fig. 2. Top: $\gamma$-ray spectra taken during beam-ON and beam OFF; bottom: time spectrum of the $803 \mathrm{keV} \gamma$-ray. The calculated time spectrum based on the known isomer half-life describes perfectly well the measured decay data (from ref. [13]).

neutron producing target. The distances between the neutron source and the uranium sample or the radiation detector were $45 \mathrm{~cm}$ and $50 \mathrm{~cm}$, respectively. For $\gamma$-ray measurements a high-purity germanium detector with $45 \%$ efficiency relative to NaI was used. The germanium crystal was n-doped to reduce the effect of fast neutron-induced radiation damage. Between the detector and the neutron source a copper cone was mounted as shielding for the detector crystal against fast neutrons. The optimum position of the shielding was controlled by using a NE213 liquid scintillation detector. Pulse-shape discrimination was employed for an efficient $\mathrm{n} / \gamma$ discrimination. Residual neutrons still reaching the detector were monitored by inspecting the well-known $\gamma$-ray line from inelastic scattering of neutrons in ${ }^{72} \mathrm{Ge}$ with $\mathrm{E}_{\gamma}=693.4 \mathrm{keV}$ during the experiment. The incident neutron energy, $\mathrm{E}_{n}$, was about $1 \mathrm{MeV}$. For the time being, only data taken with $5 \mathrm{kHz}$ repetition frequency have been analysed. The analysis of the beam-OFF spectra was carried out in three steps,

1. correction for the sample activity

2. search for $\gamma$-ray lines, which fulfil the following conditions: they appear only in the OFF-spectra, having an energy well below $\mathrm{E}_{I I}$ and fit into the decay scheme above the normal ground state

3. comparison of line intensities A1 and A2 for two different time bins for a possible half-life determination according to $\mathrm{T}_{1 / 2} \sim \ln (\mathrm{A} 2 / \mathrm{A} 1)$.

Details about the experimental set-up and the data analysis may be found in refs. $[10,17]$.
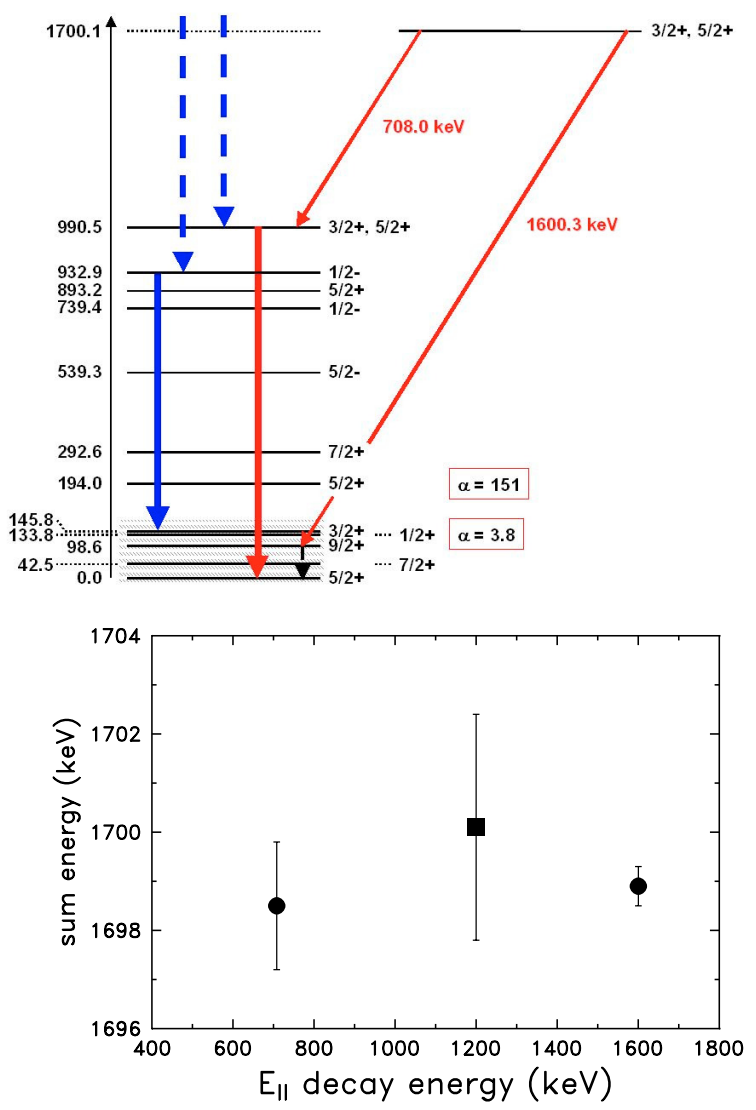

Fig. 3. Top: part of the level scheme above the normal ground state in ${ }^{239} \mathrm{U}$ for level energies $\epsilon<\mathrm{E}_{I I}=1700.1 \mathrm{keV}$. Full lines indicate presumable $\gamma$-decay of the shape isomeric ground state in ${ }^{239} \mathrm{U}$, whereas the dashed lines indicate decay above the normal ground state due to prompt neutron capture (see text). Both isomer-decay chains have the same total decay energy (circles) and correspond to the previously determined isomeric ground state energy $\mathrm{E}_{I I}=$ $1.7 \mathrm{MeV}[8,9]$.

In the upper part of figure 3 a part of the level scheme in ${ }^{239} \mathrm{U}$ is shown. At least two $\gamma$-ray lines were found depopulating the SD ground state and feeding well-known states above the normal ground state. In one case the sub-sequent $\gamma$-decay to the ground state has been observed, too. The lower part of figure 3 shows the sum-energy of both decay chains, both nicely corresponding to the observed SD ground state energy $\mathrm{E}_{I I}$. The effect of residual low-energy neutron background is visible through $\gamma$-rays de-populating levels above $\mathrm{E}_{I I}$ (dashed lines in the level scheme in fig. 3). From the presently analysed data a half-life according to step 3 could not be determined, yet.

\subsection{The fission isomer in ${ }^{235} \mathrm{U}$}

For the compound nucleus ${ }^{235} \mathrm{U}$ no fission isomer had been reported to date. Existing fission half-life estimations range between 1 and $10 \mathrm{~ms}$. Therefore, ${ }^{235} \mathrm{U}$ is one of the most favourable candidates to perform a shape-isomer search.

The fission fragments were detected with a twin Frischgrid ionisation chamber (IC) with common anode. A $1.364 \mathrm{mg}$ sample of ${ }^{234} \mathrm{U}$ was placed in the centre of the cathode 

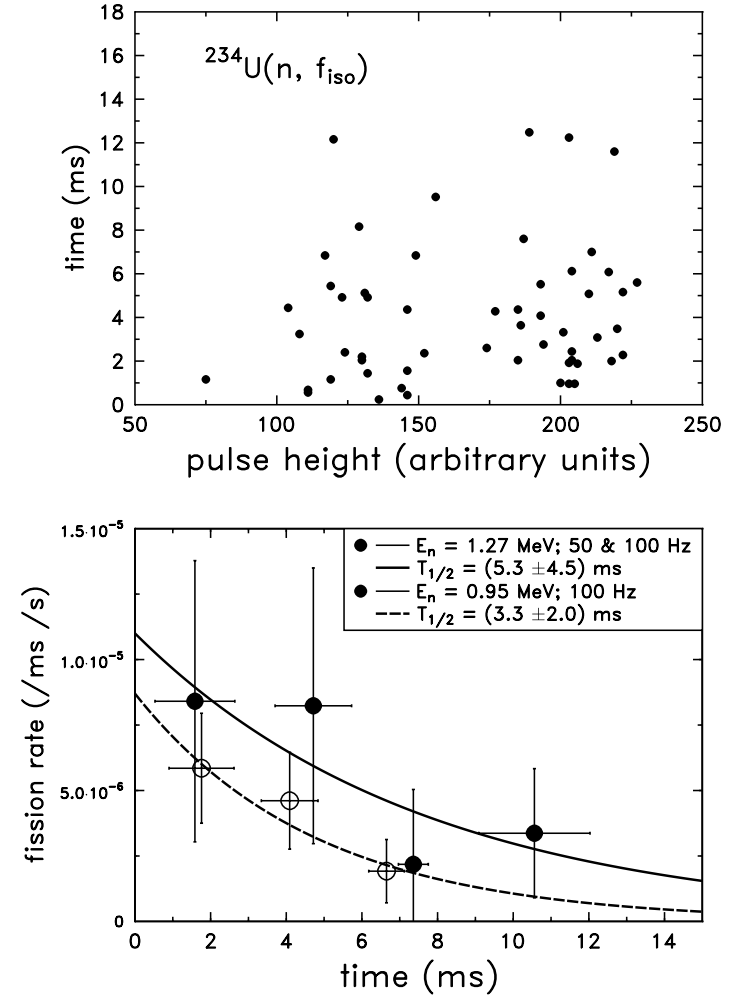

Fig. 4. Fission fragments from the decay of the shape isomer in ${ }^{235} \mathrm{U}^{*}$ : top: decay time relative to the end of teh neutron pulse vs angulardependent energy-loss corrected fission fragment pulse-height; bottom: shape-isomeric decay time distribution of ${ }^{235} \mathrm{U}$, summed in bins of $3 \mathrm{~ms}$ and background corrected. The weighted average half-life is $\mathrm{T}_{1 / 2}=(3.6 \pm 1.8) \mathrm{ms}[18]$.

closest to the neutron source. The contamination from ${ }^{235} \mathrm{U}$ was smaller than $9 \times 10^{-4}$. A sample with $2.38 \mathrm{mg}$ of ${ }^{235} \mathrm{U}$ served as monitor for room-scattered neutrons down to thermal energies and was mounted on the second cathode of the IC. Measurements were performed at $\mathrm{E}_{n}=0.95$ and $1.27 \mathrm{MeV}$ at pulse frequencies $v=100 \mathrm{~Hz}$ and 50,100 and $150 \mathrm{~Hz}$, respectively.

Possible background may consist of three components: firstly, thermalised neutrons, which have lifetimes comparable with the investigated time interval, might have induced fission in the ${ }^{235} U$ admixture in the ${ }^{234} U$ target; secondly, a time-independent background and, finally, a time-dependent background due to fast-neutron induced fission, in case that the proton beam was not perfectly deflected. Fission events induced in the ${ }^{235} \mathrm{U}$ monitor sample were taken to correct the delayed fission time spectrum bin-wise for the contribution of the ${ }^{235} \mathrm{U}$ content in the ${ }^{234} \mathrm{U}$ sample. No time-dependent neutron background was observed, and the time-independent background may be attributed to cosmic rays and results in at most one possible fission induced in the ${ }^{234} \mathrm{U}$ sample during one week of measurement [18].

The fission fragment pulse-height was measured and corrected for angular-dependent energy loss. The quality of the corrections may be depicted from the decay time relative to the end of the neutron pulse vs angular-dependent energyloss corrected fission fragment pulse-height as shown in the top part of figure 4. From the time distribution of delayed fission events collected in time bins of $3 \mathrm{~ms}$ (see bottom part of fig. 4) a decay half-life $\mathrm{T}_{1 / 2}=(3.6 \pm 1.8) \mathrm{ms}$ has been determined. From integrating the fitted Exponentials the population probability of the SD ground state in a neutroninduced reaction was determind relative to the number of prompt fissions to $\mathrm{P}_{\text {iso }}=(7.5 \pm 6.0) 10^{-6}$. This corresponds to a production cross section of $(10 \pm 8) \mu \mathrm{b}$.

\section{Summary and outlook}

Different experiments on isomer decay were successfully performed with the NEPTUNE spectrometer of the EC-JRC IRMM. The feasibility of measuring $(n, x n)$ cross sections in the presence of spin isomers was demonstrated. First experimental evidence for de-excitation of a SD shape isomer by $\gamma$ ray emission in ${ }^{239} \mathrm{U}$ was found. With ${ }^{235} \mathrm{U}$ first experimental confirmation of a shape-isomer in odd-A uranium isotopes was obtained. A measurement of the $(\mathrm{n}, \mathrm{xn})$ cross section in ${ }^{207} \mathrm{~Pb}\left(\mathrm{~T}_{1 / 2}=805 \mathrm{~ms}\right)$ as well as the search for the shape isomer in ${ }^{237} \mathrm{U}$ is planned next.

Two of the authors (A.O. and M.G.) are indebted to the European Commission for funding expenses through the Transnational Access project NUDAME ("Neutron Data Measurements at IRMM" contract No. FP6-516487) within the Sixth Framework Programme of EURATOM for performing an experiment at IRMM.

\section{References}

1. V.M. Strutinsky, Nucl. Phys. A 81, 1 (1966).

2. H. Weigmann, Zeit. Phys. 214, 7 (1968).

3. S. Björnholm, J.E. Lynn, Rev. Mod. Phys. 52, 725 (1980).

4. B. Singh, R. Zywina, R.B. Firestone, Nucl. Data Sheets 97, 241 (2002).

5. D. Pansegrau et al., Phys. Lett. B 484, 1 (2000).

6. J. Kantele et al., Phys. Rev. Lett. 51, 91 (1983), J. Kantele et al., Phys. Rev. C 29, 1693 (1984).

7. U. Goerlach et al., Phys. Rev. Lett. 48, 1160 (1982).

8. S. Oberstedt, F. Gunsing, Nucl. Phys. A 589, 435 (1995).

9. S. Oberstedt, F. Gunsing, Nucl. Phys. A 636, 129 (1998).

10. Exploratory Research at IRMM 2004, Final Report, Comp. by the IRMM Scientific Committee, Internal Report GE/SCIRMM/ER/2005 (2005).

11. GBS-Elektronik GmbH, http://www.gbs-elektronik.de.

12. EXFOR experimental data library, JANIS version 2.1 (2004).

13. A. Pavlik et al. (2006) (private communication).

14. H. Weigmann, J.P. Theobald, Nucl. Phys. A 187, 305 (1971).

15. V. Metag (1974), cited in ref. [3].

16. R. Zhongzhou, Chang Xu, Nucl. Phys. A 759, 64 (2005).

17. S. Oberstedt et al. (to be published).

18. A. Oberstedt et al. (submitted for publication). 